\title{
HAT-South: A Global Network of Southern Hemisphere Automated Telescopes to Detect Transiting Exoplanets
}

\author{
G. Bakos ${ }^{1}$, C. Afonso ${ }^{2}$, T. Henning ${ }^{2}$, A. Jordán ${ }^{2}$, M. Holman ${ }^{1}$, \\ R. W. Noyes ${ }^{1}$, P. D. Sackett ${ }^{3}$, D. Sasselov ${ }^{1}$, Gábor Kovács ${ }^{1}$, \\ Z. Csubry ${ }^{1}$, A. Pál ${ }^{1}$ \\ ${ }^{1}$ Harvard-Smithsonian Center for Astrophysics, \\ 60 Garden street, MA02138, USA \\ email: gbakos@cfa.harvard.edu \\ ${ }^{2}$ Max Planck Institute für Astronomie \\ ${ }^{3}$ Australian National University
}

\begin{abstract}
.
HAT-South is a network of six identical, fully automated wide field telescopes, to be located at three sites (Chile: Las Campanas, Australia: Siding Springs, and Namibia: HESS site) in the Southern hemisphere. The primary purpose of the network is to detect and characterize a large number of extra-solar planets transiting nearby bright stars, and to explore their diversity. Operation of HAT-South is a collaboration among the Harvard-Smithsonian Center for Astrophysics (CfA), Max Planck Institute for Astronomy (MPIA) and the Australian National University (ANU). The network is expected to be ready for initial science operations in 2009 . The three sites will permit near round-the-clock monitoring of selected fields, and the continuous data-stream will greatly enhance recovery of transits. HAT-South will be sensitive to planetary transits down to $R \approx 14$ across a 128 square-degrees combined field of view, thereby targeting a large number of dwarfs with feasible confirmation-mode follow-up. We anticipate a yearly detection rate of approximately 25 planets transiting bright stars.
\end{abstract}

\section{The HAT-South Instrument}

Instrument parameters for HAT-South (aperture, field of view, detector pixel size, quantum efficiency, optical bandpass, pointing and tracking capability) were optimized for maximum rate of detection of transiting extrasolar planets, given the scale of this effort. The so-called $\mathrm{TH}_{4}$ (Fig. 1) will carry a suite of four $0.18 \mathrm{~m} \mathrm{f} / 2.8$ Takahashi hyperbolic astrographs, each incorporating an Apogee $4 \mathrm{~K} \times 4 \mathrm{~K}$ CCD detector with Sloan $r$ filter, and each spanning a field of view (FOV) $4^{\circ} \times 4^{\circ}$. One $\mathrm{TH}_{4}$ instrument will thus have a mosaic FOV of $8^{\circ} \times 8^{\circ}$. The pixel scale will be $3^{\prime \prime}$ pixel ${ }^{-1}$. This will greatly help in eliminating diluted optical triples, a common type of false alarm that mimicks planetary transits. Each of the three sites will operate two $\mathrm{TH}_{4}$ instruments, for increased sky coverage and redundancy in case of failure. All $\mathrm{TH}_{4}$ components will be protected by a clamshell dome, and will be operated in a fully automated manner, similar to the HATNet instruments (Bakos et al. 2002, Bakos et al. 2004). 


\section{Rationale and feasibility of HAT-South}

\subsection{Why a global network?}

Nearly "round-the-clock" monitoring of stars greatly increases detectability of transiting planets, particularly those with periods in excess of a few days or far from integer fractions of a day. Weather permitting, for an anti-solar field at suitable declination we can gather a 24-hour continuous data-stream, with the exception of brief interruptions during summer nights. Fig. 2 shows the recovery function originating from a realistic simulation that was performed having the same number (i.e. a total of 6) instruments on a single site (Chile), observing the same field, equally spread over all combinations of two sites (3 at each site), or over three sites (Namibia:2, Chile:2, Australia:2). For the ease of combining data originating from the different sites, we use truly identical instruments.

\subsection{Why a medium-depth survey?}

Existing transit surveys generally have a bi-modal distribution in magnitude range; shallow (SH) surveys, such as HATNet, are sensitive down to $R \lesssim 12$, whereas deep (D) surveys are sensitive at $R \gtrsim 14$. HAT-South will bridge most of the gap in between with a medium-depth (MD) survey $(9.0 \lesssim R \lesssim 14)$. This choice is useful for three reasons: (i) the ideal stars for harboring detectable transiting planets are F0-M5 dwarfs, whose mean density on the sky for an MD survey is an order of magnitude greater than for an $\mathrm{SH}$ survey when integrated over the respective magnitude ranges. (ii) The ratio of dwarfs to giants increases rapidly toward higher Galactic latitudes, where for an MD survey it is about 9, whereas for an SH survey it is only about 3; this significantly eases the problem of dealing with "false positive" detections due to contamination by giants. (iii) The ratio of late, small-radius dwarfs to F \& G dwarfs also increases in an MD survey; this facilitates detection of smaller planets. At the same time, the magnitude range covered

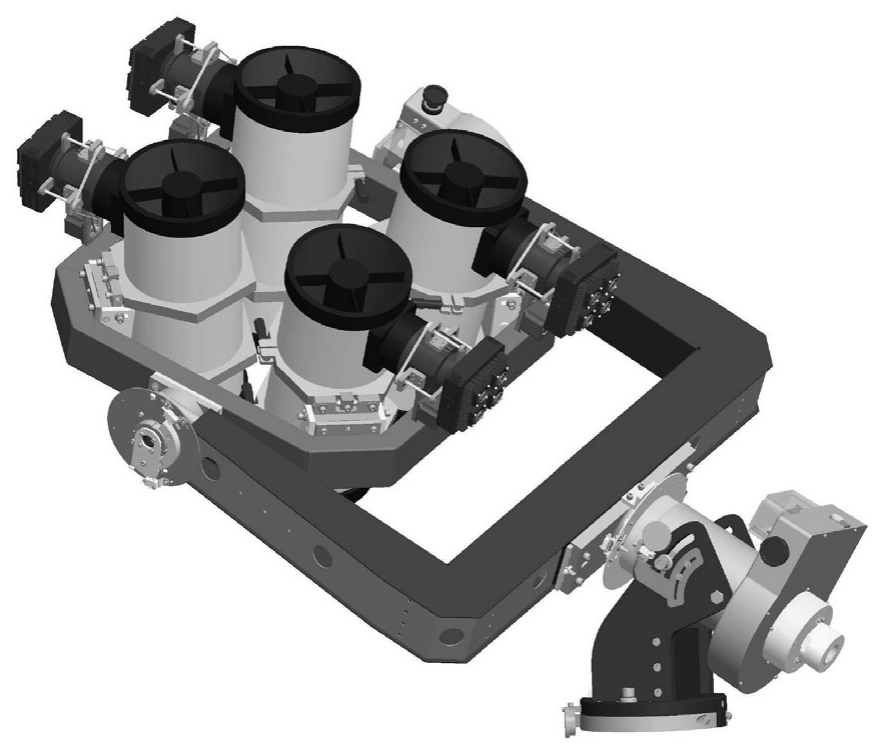

Figure 1. Engineering model of HAT-South. A forkmount holds four telescope tubes, each $0.18 \mathrm{~m}$ diameter with $0.5 \mathrm{~m}$ focus, holding a $4 \mathrm{~K} \times 4 \mathrm{~K}$ CCD yielding a combined FOV of $8^{\circ} \times 8^{\circ}$. 


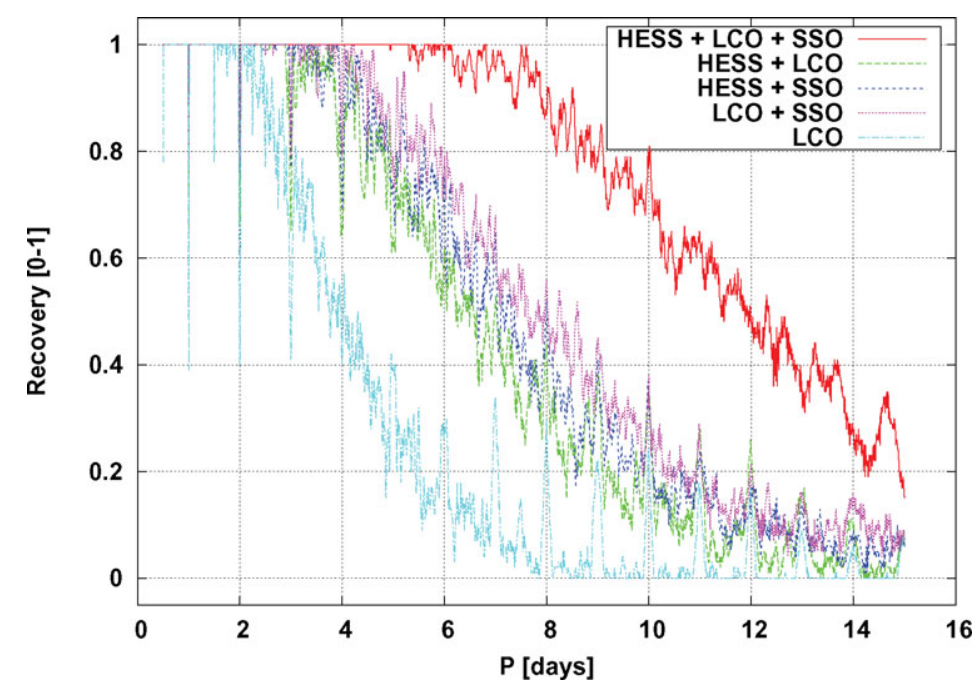

Figure 2. Transit recovery as a function of period for different site configurations. Top curve shows a 3 site simulation with 2-month observing window during the summer (short nights). The curves in the middle show 2 site configurations (3 possibilities) for the same time-frame. The bottom curve is for a single site. Further assumptions: known $65 \%$ clear weather statistics, an estimated $15 \%$ instrument down-time, $1 \%$ white-noise, $0.1 \%$ red-noise, and $1 \%$ transit depth; transit detected if at least 3 transits observed, each covering at least $20 \%$ of the transit duration, and with Box Least Squares (BLS) SNR $>7$.

by the medium-depth HAT-South is bright enough for spectroscopic confirmation with moderate telescopes. Altogether, the MD survey range represents a unique "sweet spot" for detection, confirmation, and follow-up of transiting planets.

\subsection{Why the southern hemisphere?}

South America, Africa, and Australia are roughly equally spaced in longitude, plus they have well-developed observing sites with excellent astroclimate.

The southern hemisphere presents another advantage over the north: due to the structure of the Galaxy, the number density of ideal planet-host stars down to $R<14$ (the approximate sensitivity limit with HAT-South) at e.g. $b_{\text {gal }} \approx 20^{\circ}$ is $1.5 \times$ higher at $l \approx 0^{\circ}$ (towards the Galactic Center; GC) compared to $l \approx 180^{\circ}$, and because the visibility of the GC is better from the South (overhead in the winter), there are more ideal fields present for transit searches.

Also, the southern hemisphere has excellent facilities for spectroscopic confirmation of planet candidates, as described below in $\S 2.5$.

\subsection{Feasibility tests}

We have performed thorough tests to demonstrate the attainable photometric precision of the proposed setup using a U16m CCD on our existing TopHAT mount and Takahashi BRC-250 optics to obtain a 2-night sequence of 120 -sec exposures. The results confirmed that $1 \%$ precision can be reached at $R \approx 14$ using 4 -minute cadence. Later we carried out observations for one week from FLWO using the final setup with an Takahashi Epsilon astrograph optics, mounted on the TopHAT mount, and using the U16m CCD, i.e. all critical components being identical to the final hardware setup of HAT-South. The results showed that the final system will fully meet expectations. The prototype design has been 
finalized, and the mechanical and electronics part of the mount and dome are being manufactured.

\subsection{Follow-up Observations}

Based on our HATNet experience, we expect HAT-South to yield of order 250 planet candidates per year, so that we will be faced with the task of culling that sample to detect the approximately 25 true transiting planets.

Once a candidate transiting planet host is identified, it is important to obtain reconnaissance spectroscopy. Such spectra can yield an estimate of the star's effective temperature, surface gravity, rotation rate $v \sin (i)$, and radial velocity variation. Examples of the instruments we might use for this purpose are (with telescopes in parantheses): Coralie (Euler $1.5 \mathrm{~m}$ at La Silla), FEROS (La Silla $2.2 \mathrm{~m}$ ), du Pont Echelle (du Pont 2.5m), UCLES (4m AAT), ANU Echelle (2.3m ANU).

If a candidate survives reconnaissance spectroscopy, and if the HAT-South transit signal is sufficiently robust, we may proceed directly to high SNR spectroscopy, using an instrument capable of velocity precision of a few $\mathrm{m} / \mathrm{s}$ or better. Examples of such instruments expected to be available to our combined team are: HARPS (La Silla 3.6m), Coralie (Euler $1.5 \mathrm{~m}$ in La Silla), UVES (8m VLT), UCLES (4m AAT), HARPS-N (4m WHT).

\section{Expected Results and Scientific Impact}

Our analysis of Besançon models of the Galaxy (Robin et al. (2003)) indicate about 230 F5-M5 dwarfs per $\square^{\circ}$ with $R<14$, or 30000 in the combined $128 \square^{\circ}$ HAT-South fields at $b \approx 20^{\circ}$. Gaudi (2006) estimates 1 in 500 of dwarf stars are accompanied by close in $(\mathrm{P}<5$ days $)$ giant planets, of which about $10 \%$ would have orbital inclinations leading to transits. Thus we expect about 6 detectable planets in the combined field. This is probably an underestimate, since it does not consider longer-period planets for which HAT-South will have efficient recovery rates. We conservatively use the 3 -site simulation of Fig. 2 for a 1-month observation window during summer time (short nights), taking into account weather statistics and instrument downtime. Based on this we adopt what we believe to be an overly pessimistic mean transit recovery rate of only $40 \%$; this is much smaller than shown in the figure. Thus we predict minimum recovery of $\mathbf{2}$ planets per month or about 25 per year. Thus in the five years of the proposed program, HAT-South should detect and characterize of order 125 transiting exoplanets.

\section{Acknowledgements}

HAT-South instrumentation was supported by the National Science Foundation (NSF) Major Research Instrumentation (MRI) program, grant number AST-0723074, and also Smithsonian Astrophysical Observatory internal research grants. G. Bakos is a National Science Foundation Fellow, supported by grant AST-0702843.

\section{References}

Bakos et al. 2002, PASP, 114, 974

Bakos et al. 2004, A\&AP, 116, 266

Gaudi, S., 2006, ASP Conf. Ser., astroph/12141

Robin et al. 2003, A\&AP, 409, 523 